\title{
Buffer-gas Loading and Magnetic Trapping of Molecules
}

\author{
John M. Doyle ${ }^{1,2}$ \\ ${ }^{1}$ Department of Physics, Harvard University \\ ${ }^{2}$ Harvard-MIT Center for Ultracold Atoms
}

\begin{abstract}
Ultracold and cold molecules have enormous potential for yielding a wealth of new physics and new technologies. Molecules possess several features that greatly increase the possibilities for new interactions, collective quantum states and effects, collisional processes, fundamental symmetry tests and chemical processes. This is due to 1) the strong interactions between the dipole moments of polar molecules, 2) the rotational and vibrational internal structure of all molecules, and 3) the easily orientable internal electric field of many molecules. The promise of cold and ultracold molecules will only be realized when samples can be prepared with at least the same ease in which we now prepare atomic samples. Several approaches towards trapping of molecules, the key first step toward studying ultracold molecules at high density, have already succeeded: direct cooling of molecules via a buffer gas, mechanical slowing of a pulsed molecular beam with electric fields and photoassociation of alkali atoms. In the ICAP 2002 session on molecules I described some of the current theoretical results and predictions about ultracold molecular systems. Profs. Meijer and Heinzen described results on pulsed beam slowing and photoassociation. This contribution describes some of the key results on buffer-gas loading of molecules.
\end{abstract}

\section{A. Introduction}

The ability to cool and trap atoms set atomic physics on a new course marked by milestone experiments such as the observation of Bose-Einstein condensation (BEC) [1], Fermi degeneracy [2] in a gas, superfluidity in atomic vapors [3], atom lasers [4], and non-linear atom optics [5]-[7]. The challenge in molecular physics is to achieve with molecules what has so far been limited to atoms. This prospect is perhaps even more appealing, since molecules offer a vast range of properties not available with atoms. Unlike atoms, molecules are generally non-spherical and have rotational and vibrational degrees of freedom. Apart from that, molecules possess both even and odd multipole moments - electric and magnetic - that are all coupled to the rotating molecular frame. As a result, any molecular interaction, whether with other molecules or with external fields, is shaped and refined by a transformation between the rotating molecular frame and the space-fixed laboratory frame.

Cooling and trapping of molecules can produce a number of significant benefits for a wide variety of applications. Availability of reduced line broadening and long interaction times can benefit precision spectroscopy [8], and coherent control [9]. Cooling of internal molecular states, combined with long interaction times, can greatly increase the sensitivity of certain precision measurements [10]. Low translational energy of the cooled molecules will facilitate external-field manipulations of molecular trajectories and orientations [11]-[13]. In the regime where the deBroglie wavelength exceeds molecular dimensions, reactive collisions start exhibiting significant quantum effects [14]. Reactive and non-reactive collisions of ultracold molecules are of significant interest [15],[16]. Beyond collisional properties, proposals exist for employing electric dipoles of ultracold molecules to implement quantum computing [17]. Finally, achiev- 
ing quantum degeneracy with molecules remains a prominent goal. It will greatly expand the scope for study of collective quantum phenomena. For example, ensembles of polar molecules would constitute a system of relatively strongly interacting particles. In a fermi-degenerate gas of polar molecules, the electric dipole-dipole interaction, which is predicted to be collision energy independent [18]-[21], may give rise to a molecular superfluid via BCS pairing. Bose condensation in dipolar gases is also predicted to yield some very interesting behavior[22]-citebaranovbec.

\section{B. Cooling of Molecules - Overview of Experimental Work}

Laser cooling and evaporative cooling have been the quintessential techniques for producing ultracold atoms. Evaporative cooling may be expected to work with molecules as well, provided their (yet unknown) elastic and inelastic low-temperature collisional cross sections are similar to those of atoms. Laser cooling of molecules, however, appears quite difficult, as the requisite closed-cycle transitions are generally not available due to the complex rovibrational level structure [26]. Four alternative techniques of cooling molecules, based on other principles, have been successfully implemented: (1) Buffer-gas cooling relies on thermalization of molecules with a cold He-buffer gas; about $10^{8} \mathrm{CaH}$ molecules, produced by laser ablation inside a buffer-gas cell, were cooled to $400 \mathrm{mK}$ and confined in a magnetic trap [27]; (2) Stark decelaration takes advantage of the adiabatic increase of Stark energy of polar molecules in an electrostatic field; up to $10^{8} \mathrm{CO}$ or $\mathrm{ND}_{3}$ molecules produced by a pulsed supersonic expansion were slowed down to a standstill and confined in an electrostatic trap at a temperature of about $30 \mathrm{mK}$ [28] and, more recently, in a storage ring [29]; (3) Photoassociation of ultracold alkali atoms has been demonstrated to produce up to $10^{6}$ dialkali molecules at temperatures in the $\mu \mathrm{K}$ range [30]-[32]; (4) Supersonic expansion from a counterrotating nozzle has been demonstrated to produce $9 \mathrm{~K}$ molecules in the laboratory frame [33]. Also, slowing molecules using a laser scoop has been recently proposed $[34],[35]$.

Buffer gas cooling is a very powerful technique. It is versatile and applicable to any atom or molecule, since it relies solely on elastic scattering. In addition, cooling of the translational degrees of freedom in the buffer gas is accompanied by efficient rotational cooling [36].

\section{Buffer-gas Loading}

Over the past three years we have developed a cooling technique that is applicable to a wide range of species, including molecules. This technique relies on thermalization with a cold He buffer gas via elastic collisions. The collisional cross section of atoms and molecules with $\mathrm{He}$ is independent of any particular energy level pattern and can increase with decreasing temperature, thus lending the technique the desired generality and efficiency.

In our work, we combined buffer-gas cooling with magnetic trapping. First we demonstrated the technique by buffer-gas loading atomic europium (with a maximum dipole moment of 7 Bohr magnetons) into a magnetic trap. This work corroborated the feasibility of the scheme and produced the first benefits of magnetic trapping of a complex atom: a hyperfine resolved Zeeman spectrum whose variation with time provided clues about the relaxation dynamics of the trapped states. About $10^{12} \mathrm{Eu}$ atoms were trapped.

Next, we buffer gas loaded isotopically pure chromium atoms, ${ }^{52} \mathrm{Cr}$ (a 6 Bohr magneton species). Like the $\mathrm{Eu}$ atoms, $\mathrm{Cr}$ atoms were prepared by laser ablation of a solid metal precursor. More than $10^{11} \mathrm{Cr}$ atoms were trapped. More recent work 


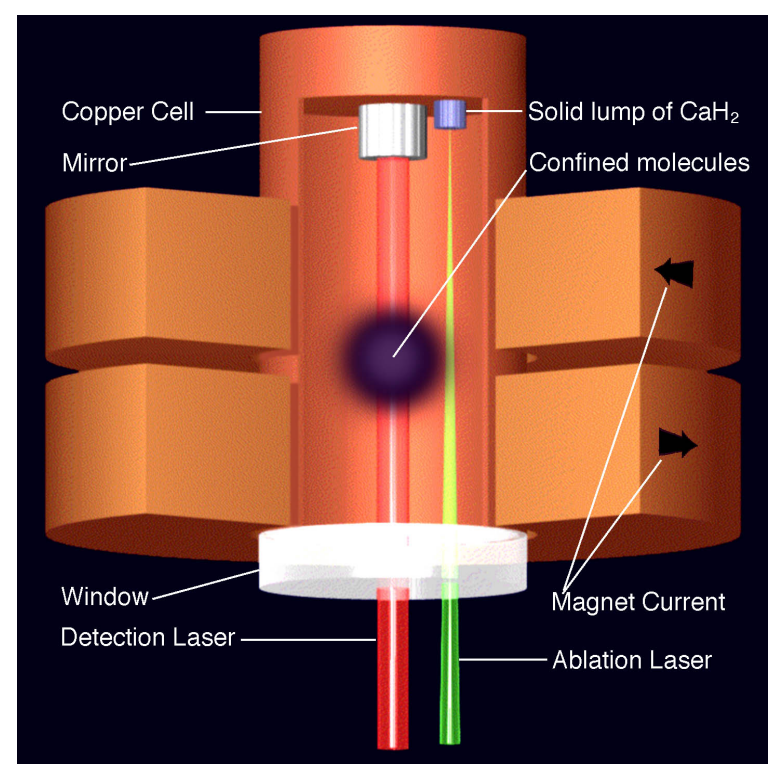

Figure 1: Section through the core elements of the buffer-gas loaded magnetic trap.

has shown the evaporative cooling of bosonic and fermionic $\mathrm{Cr}$ and the discovery of interesting structure in its inelastic collision cross section. [37, 38].

Our efforts towards molecular trapping culminated in the trapping of $\mathrm{CaH}$. The molecules (with a magnetic dipole moment of 1 Bohr magneton) were prepared by laser ablation of a solid $\mathrm{CaH}_{2}$ precursor. About $10^{8}$ were loaded using ${ }^{3} \mathrm{He}$-buffer into an anti-Helmholtz magnetic trap. Detailed, time dependent Zeeman spectra were measured using laser induced fluorescence spectroscopy. These spectra provided insights into both the trapping dynamics and the electronic structure of $\mathrm{CaH}$.

\section{Apparatus}

The cross-section of the cryogenic apparatus we have used in our molecular trapping of $\mathrm{CaH}$ (and earlier atom trapping) experiments is depicted in Figure 1. The apparatus consists of three main parts: the superconducting magnet, the cryogenic cell, and the dilution refrigerator. The magnet $(5.1 \mathrm{~cm}$ clear bore) consists of two $\mathrm{NbTi}$ superconducting solenoids encased in a titanium cask. The two coils are arranged in the anti-Helmholtz configuration and are immersed in liquid helium.

The cell is positioned at the center of the magnet. It resides in vacuum and is separated from the magnet (and the liquid helium) by a stainless steel vacuum can, a tube of $5 \mathrm{~cm}$ diameter and $0.8 \mathrm{~mm}$ wall thickness. The cell is made of OFE copper with a $4.4 \mathrm{~cm}$ diameter fused silica window sealing the bottom. On the inner top surface there is a $1 \mathrm{~cm}$ diameter mirror. A solid lump of the precursor material is positioned near the mirror. The top of the cell is thermally anchored to the mixing chamber of the dilution refrigerator. The temperature of the cell can easily be varied from $100 \mathrm{mK}$ to $800 \mathrm{mK}$ using a resistive heater. 
Our detection method is either absorption spectroscopy (used mainly for atoms but also with molecules) or laser induced fluorescence (used only for molecules). A pulsed doubled YAG laser beam is used to ablate the solid precursor material.

\section{Procedure}

Our trapping procedure begins with the cryogenic cell filled either with ${ }^{4} \mathrm{He}$ or ${ }^{3} \mathrm{He}$ gas. The temperature of the cell is raised prior to ablation. The cell is heated to a temperature corresponding to the desired density $\left(\sim 10^{16} \mathrm{~cm}^{-3}\right)$ of the helium buffer gas. Then the ablation laser is fired, producing molecules that diffuse through the buffer gas and thermalize with it. After ablation, the cell temperature is lowered. This cryopumps the helium gas to the walls of the cell.

The CaH molecules are created by ablating a solid sample of $\mathrm{CaH}_{2}$ placed within the cell at the edge of the trapping region with a $10 \mathrm{~mJ}, 7 \mathrm{~ns}$ YAG pulse [40].

The CaH molecules are detected by laser fluorescence spectroscopy [40]. The fluorescence is excited at $635 \mathrm{~nm}$ in the $B^{2} \Sigma, v^{\prime}=0 \leftarrow X^{2} \Sigma, v^{\prime \prime}=0$ band [41],[42] and detected mainly within the $B, v^{\prime}=0 \rightarrow X, v^{\prime \prime}=1$ band at $692 \mathrm{~nm}$ (the corresponding Franck-Condon factor was calculated to be 0.028, [43]). A set of color-glass and band-pass interference filters placed in front of the detector (either a photomultiplier tube or a CCD camera) serves to block the scattered probe radiation (along with the $B, v^{\prime}=0 \rightarrow X, v^{\prime \prime}=0$ fluorescence).

Typically, under field free conditions, only a single rotational transition, $N^{\prime}=$ $1, J^{\prime}=3 / 2 \leftarrow N^{\prime \prime}=0, J^{\prime \prime}=1 / 2$, can be detected. This is consistent with a fast rotational relaxation of the molecules that renders the population of higher rotational states negligible: the next rotational state lies $8.5 \mathrm{~cm}^{-1}$ above the $N^{\prime \prime}=0, J^{\prime \prime}=1 / 2$ ground state and the intensity of the (unobserved) corresponding transition is well below $0.1 \%$ of the $N^{\prime}=1, J^{\prime}=3 / 2 \leftarrow N^{\prime \prime}=0, J^{\prime \prime}=1 / 2$ transition. This yields an upper limit on the rotational temperature of $1.5 \mathrm{~K}$. Using absorption spectroscopy to calibrate the fluorescence detection, we found that up to $10^{10} \mathrm{CaH}$ molecules could, under certain conditions, be formed by a single ablation pulse. We were also able to detect the formation of $X^{2} \Sigma, v^{\prime \prime}=1$ molecules, which were about $10-100$ times fewer; their scarcity precluded them from being detected in the trap. However, we measured an upper limit of the $v^{\prime \prime}=1 \rightarrow v^{\prime}=0$ relaxation cross section (in collisions with the ${ }^{3} \mathrm{He}$ buffer gas) to be $10^{-18} \mathrm{~cm}^{2}$, which suggests that it should be possible to load them into the trap.

As the magnetic field is turned up, the field-free rotational transition is observed to split into two features shifted towards lower and higher frequencies. Theoretical analysis [44] assigns the feature that shifts towards higher frequencies to the transition from the $N^{\prime \prime}=0, J^{\prime \prime}=1 / 2, M^{\prime \prime}=1 / 2$ low-field seeking state to the $N^{\prime}=1, J^{\prime}=$ $3 / 2, M^{\prime}=3 / 2$ state, and the feature that shifts towards lower frequencies to the transition from the $N^{\prime \prime}=0, J^{\prime \prime}=1 / 2, M^{\prime \prime}=-1 / 2$ high-field seeking state to the $N^{\prime}=1, J^{\prime}=3 / 2, M^{\prime}=-3 / 2$ state.

At later times after the ablation pulse, the high-field seekers move towards the edge of the trap, hit the wall of the cell, stick to it, and are lost from the trap; the lowfield seekers, on the other hand, move towards the center of the trap and by $300 \mathrm{~ms}$ their distribution is that of a trapped ensemble at $T=400 \mathrm{mK}$, close to the initial temperature of the buffer gas of $300 \mathrm{mK}$. This is displayed in Figure 2, which shows spectra taken at different time delays with respect to the ablation pulse. After about $300 \mathrm{~ms}$ the signal corresponding to the high-field seekers (right-hand peak) is absorbed by the noise, see the lowest panel of Figure 2. The final low-field-seeker distribution (last panel) fits very well to a trapped distribution with a temperature $T=400 \mathrm{mK}$ 
and a number of molecules, $N=10^{8}$.

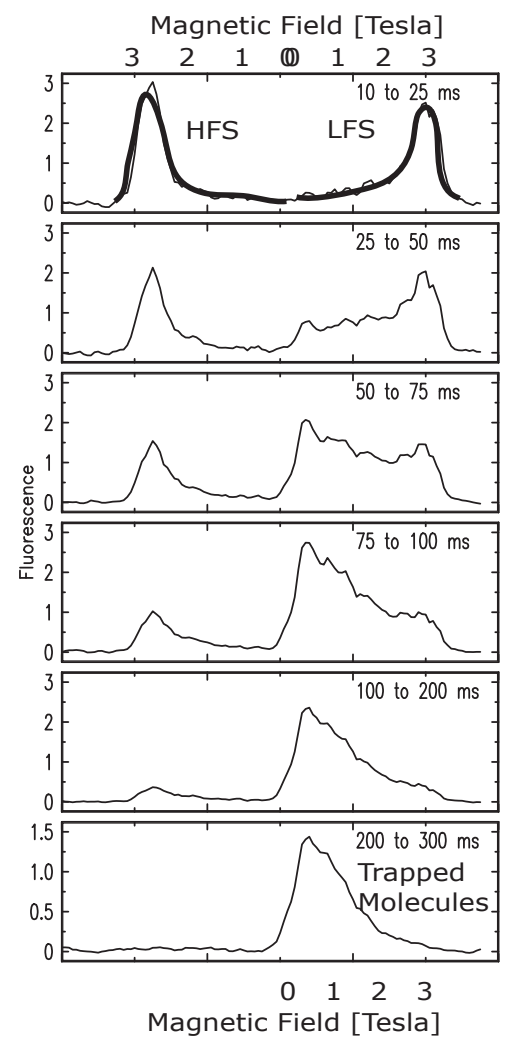

Figure 2: Time evolution of the $\mathrm{CaH}$ spectrum.

\section{Direct Beam Load-} ing

In buffer-gas work described above, laser ablation [36] was used to introduce $\mathrm{CaH}$ into the buffergas cell. Laser ablation requires a suitable solid precursor, which is not always easily found. One is often forced to rely on the breakdown of more complex precursor molecules. For certain types of precursors, rapid destruction of the precursor pellet may occur (turning the pellet into dust, "drilling" a hole into it, etc.) [10]. The ablation process usually lacks specificity and unwanted species, including clusters, often form as by-products. If these react with, or adsorb the species of interest, its population may be dramatically depleted [45]. Also, the yield of the molecules of interest per ablation pulse is limited and hard to predict (however, for certain precursors under suitable conditions, the yield per pulse can be quite constant [10]). Finally, ablation pulses bring

additional heat into the cryogenic cell.

We describe here a proof-of-principle experiment that demonstrates loading of a cryogenic buffer-gas cell maintained at $4.2 \mathrm{~K}$ with a beam of rubidium atoms and thermalization of the atoms with the buffer-gas. This beam-based method offers the following advantages: (1) It is versatile - atomic and molecular beam sources for a variety of atoms and molecules, including radicals, metastable species and roomtemperature solids, are available [46],[47]; (2) Fluxes as large as $10^{15} \mathrm{~s}^{-1}$ entering the cell can be expected from high-intensity beam sources, potentially leading to trapping of $>10^{14}$ molecules (or atoms) using buffer-gas trapping techniques [36]; (3) Beam sources make it possible to eliminate or to control the formation of clusters or other unwanted species; (4) For many gases and the low-melting point solids, sources are simple in design and inexpensive; (5) Multiple sources can be operated so as to introduce a combination of species into the buffer gas. 


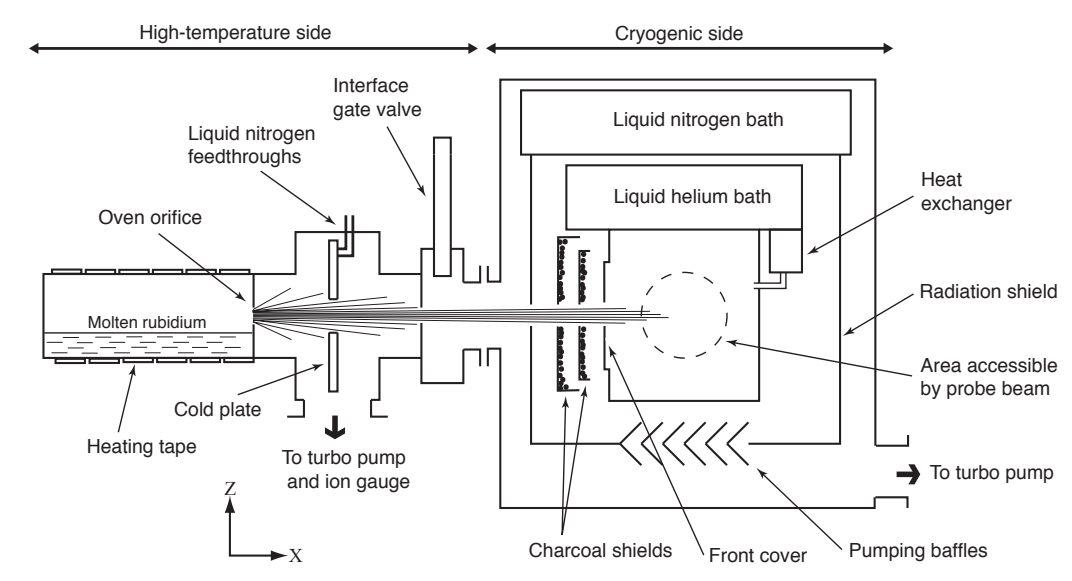

Figure 3: Schematic view of the apparatus.

\section{Experimental Setup}

The apparatus, shown in Figure 3, consists of two main parts, one cryogenic and one at high temperature. These are interfaced via a gate valve. The cryogenic part is based on a small liquid-nitrogen-shielded liquid helium cryostat with $1.2 \mathrm{~L}$ helium bath [48]. A cylindrical brass cryocell is thermally anchored to the liquid helium bath. The cryocell is $10 \mathrm{~cm}$ long, and has an outer diameter of $6 \mathrm{~cm}$ and an internal volume $V=150 \mathrm{~cm}^{3}$. The side of the cell facing the interface gate valve has a thin replaceable front cover, made out of copper, mounted on an indium seal. The front cover has a circular orifice (cell orifice) whose diameter could be varied from 0 up to $3 \mathrm{~mm}$. The molecular beam enters the cell through this orifice. At the same time, the He-buffer gas leaks out through the cell orifice. A heat exchanger, made out of copper tubing [49], is mounted on the cryostat cold plate. The outlet of the heat exchanger is connected to the cell; the inlet (at room temperature) to an external gas handling system via a $1.1 \mathrm{~mm}$ inner diameter stainless steel tube. The gas handling system supplies helium gas (99.999\% purity) at an adjustable steady rate such that the helium pressure in the cell remains constant (balanced by the helium leaving through the cell orifice). The heat exchanger ensures that the He gas entering the cell has the temperature of the liquid helium bath. The cryocell is equipped with a pair of windows which enable optical access to a cell region. The temperature of the cell is monitored by silicon diode thermometers, and the pressure inside the cell by a Pirani gauge [50].

The liquid-helium bath and the cell are surrounded by a radiation shield connected to a liquid nitrogen bath. Two charcoal shields [51] are placed between the cell orifice and the liquid nitrogen shield to pump the He which leaks out from the cell orifice. The inner shield is thermally linked to the cell, the outer shield to the liquid helium bath. Most of the leaking He atoms are pumped by the inner shield and thus cannot reach the liquid-nitrogen radiation shield; those that do are likely to hit the outer charcoal shield after they bounce back. The heat imparted to the He atoms by the 
liquid-nitrogen shield is then deposited in the liquid helium bath and does not increase the temperature of the cryocell. Charcoal saturation can be a problem for very high He-flow rates, so resistive heaters are placed on the shields to regenerate the charcoal if necessary. Under typical operating conditions, saturation does not occur for many hours, as expected [52]. When saturation occurs, the shields quickly overheat and self-regenerate, so the system is back in operation within minutes.

The high-temperature part of the apparatus consists of an oven and a collimation chamber. The front wall of the oven has a $3.9 \mathrm{~mm}$ diameter orifice. The distance between the orifice and the front of the cryocell is $25 \mathrm{~cm}$. The oven is loaded with rubidium, and heated up to about $350^{\circ} \mathrm{C}$ (measured by thermocouples). The temperature of the oven is nonuniform, with measured temperature differentials between different surface points of up to $30^{\circ} \mathrm{C}$. We used the reading of the thermocouple closest to the oven orifice as the nominal oven temperature. With increasing oven temperature, the Rb flow changes from effusive towards hydrodynamic; we use less than $10^{-3}$ sr of the beam.

The collimation chamber, located between the oven and the interface gate valve, is fitted with a connection to a turbo pump and a cooled $(160 \mathrm{~K})$ condenser plate (placed about $6 \mathrm{~cm}$ downstream from oven orifice). The plate has an $8 \mathrm{~mm}$ diameter aperture to collimate the beam. As the flux of the atoms out of the oven increases with increasing temperature, the pressure in the chamber rises above $10^{-4} \mathrm{mbar}$ (the upper limit of our gauge). We did not observe any reduction of the beam flux into the cryocell due to high background pressure in the chamber. This type of setup was used in the case of rubidium because it reacts violently, and thus it is necessary for practical reasons to prevent its accumulation in the cryostat. For many other species the differential pumping cold plate would not be necessary.

We measured the temperature and density of the $\mathrm{Rb}$ atoms in the cell using $5^{2} S_{1 / 2}, F=2 \rightarrow 5^{2} P_{3 / 2}, F^{\prime}=1,2,3$ hyperfine transitions of the strong $\mathrm{D}_{2}$ absorption line of ${ }^{87} \mathrm{Rb}$ (natural abundance of $27 \%$ ) at $780 \mathrm{~nm}$. The separations of these lines are the largest among the four $\mathrm{D}_{2}$ hyperfine triplets of both $\mathrm{Rb}$ isotopes [54], thus the overlap of Doppler-broadened lines at $4.2 \mathrm{~K}$ is avoided. The natural linewidth of the transitions of about $6 \mathrm{MHz}$ is about a factor of 10 smaller than the $4.2 \mathrm{~K}$ Doppler broadening. Thus, the translational temperature of the Rb atoms could be accurately determined from fitting the measured line profiles to Voigt line shapes. Our laser source has a typical linewidth of $1 \mathrm{MHz}$ and a Gaussian intensity profile with a $0.9 \mathrm{~mm} e^{-2}$ width. The laser beam is split among a single-mode fiber transmitting several $\mu \mathrm{W}$ to the cryocell, a wavemeter, and a saturation absorption setup used for calibrating both the wavelength and the optical density [55]. The cryostat is fitted with a platform which consists of a fiber out-coupler and a photodiode mounted on an $\mathrm{X}-\mathrm{Z}$ translation stage ( $\mathrm{X}$ is the direction of the $\mathrm{Rb}$ beam, $\mathrm{Y}$ the direction of the probe laser beam). This allows the probe beam to address points inside the cell anywhere within the $30 \mathrm{~mm}$ diameter cell window. The laser frequency can be scanned over $1 \mathrm{GHz}$. The photodiode signal was averaged typically over 30 scans per data point. Baseline subtraction was accomplished by blocking the Rb beam. The sensitivity of the absorption measurement was better than $10^{-3}$.

\section{Density distribution over the cryogenic cell}

Spectroscopic mesurements of the doppler profile idicate the Rb are thermalized with the buffer gas. Now, the absorption signal is proportional to the integral of rubidium number density along the laser beam, i.e. along the Y-axis. To convert the signal into local rubidium densities or into the total number of atoms loaded, 
it is necessary to adopt a model of rubidium density distribution. A Monte-Carlo simulation of elastic collisions gives an accurate description of the loading process, however, due to significant computing time requirements it was used to process only a small fraction of the data. From our simulations we found that the range of input parameters that describes the optimal buffer-gas density corresponds to atoms entering the cell and thermalizing within several millimeters from the opening, and then diffusing across the cell into the probed volume. This behavior can be approximated by a diffusion equation with boundary conditions corresponding to absorption of particles at the walls, which is then used to convert the absorption data into number densities. At the (optimal) buffer-gas density of $n_{\mathrm{He}}=1.5 \times 10^{16} \mathrm{~cm}^{-3}$, Rb density of $n_{\mathrm{Rb}}=(8 \pm 4) \times 10^{9} \mathrm{~cm}^{-3}$ at the center of the cell is observed, indicating a total number of thermalized $\mathrm{Rb}$ atoms loaded into the cell of $N_{\mathrm{Rb}}=(1 \pm 0.5) \times 10^{12}$. To determine $F_{c}$, the flux of Rb atoms entering the cell with no buffer gas present, we scanned the probe beam across the atomic beam inside the cell, and converted the absorption signal into $F_{c}$. In doing so, we took into account the Gaussian intensity profile of the laser beam and cylindrical symmetry of the atomic beam. We then introduced buffer gas into the cell, and measured the corresponding $N_{\mathrm{Rb}}$. Due to tecnical limitations, we were able to perform this measurement only at low fluxes, up to the level $F_{c}=5 \times 10^{11}$ $\mathrm{s}^{-1}$, for which $N_{\mathrm{Rb}}=1.5 \times 10^{10}$. We assume that with increasing flux of Rb atoms $N_{\mathrm{Rb}}$ should not increase more rapidly than $F_{c}$. We therefore estimate the highest flux achieved in our experiment to be at least $F_{c}=(3 \pm 1.5) \times 10^{13} \mathrm{~s}^{-1}$.

Based on the value for Rb-He diffusion coefficient in Ref. [57] we estimate the scattering cross-section, $\sigma$, at $4.2 \mathrm{~K}$ to be $0.5 \mathrm{~nm}^{2}$, which gives the diffusion time $\tau$ $\approx 100 \mathrm{~ms}$. For $\tau=100 \mathrm{~ms}$ and $N_{\mathrm{Rb}}=1.5 \times 10^{10}$ we calculate the number of Rb atoms entering the cell with the buffer gas present as $\dot{N}_{\text {in }}=1.5 \times 10^{11} \mathrm{~s}^{-1}$. This number is approximately 3 times smaller than the measured number of atoms entering the cell with no buffer gas. This indicates that a significant fraction of $\mathrm{Rb}$ atoms coming towards the cell orifice are loaded into the buffer gas.

\section{E. Prospects}

Direct loading of a molecular beam into a cryogenic buffer-gas cell is a facile and efficient technique of cooling gaseous species. The apparatus described can be used to carry out a variety of spectroscopic measurements on atoms, molecules, radicals, and metastable species at cryogenic temperatures. The apparatus can be extended by adding another molecular beam, to study cold collisions and chemical reactions.

An exceptionally wide variety of atomic and molecular species can be produced in a molecular beam [46],[47]. Since our technique makes no use of cooling due to the beam expansion, the only parameter of interest is the beam flux in the forward direction. As a result, a simple effusive source with modest pumping requirements may suffice; the stagnation pressure can, of course, be significantly increased if greater flux is required. Since the beam temperature is insignificant in our scheme, the source can be operated at as high a temperature as necessary for raising the vapor pressure or for enabling the generation of the species of interest. As a result, any species that can form a molecular beam can be loaded into the cryogenic cell, including reactive species such as radicals.

The technique could also be used to load a magnetic trap enclosing the cell. We expect to be able to load and magnetically trap a large number of paramagnetic molecules. By applying evaporative cooling, the relatively high loading temperatures (between $0.24 \mathrm{~K}$ and $1.2 \mathrm{~K}$ ) could then be reduced to the $\mathrm{mK}$ range or lower. An experiment is underway to trap $\mathrm{NH}\left(\mathrm{X}^{3} \Sigma^{-}\right)$molecular radicals; we expect to load 
about $10^{12} \mathrm{NH}$ molecules into a He-buffer gas at $1.2 \mathrm{~K}$, and then magnetically trap over $10^{11}$ of them. As the technique is simple and versatile, many other applications are likely to emerge.

\section{References}

[1] M. Anderson, J. Ensher, M. Matthews, C. Wieman, and E. Cornell, Science 269, 198 (1995).

[2] B. de Marco and D. Jin, Science, 285, 1703 (1999).

[3] R. Onofrio, C. Raman, J. M. Vogels, J. Abo-Shaeer, A.P. Chikkatur, and W. Ketterle, Phys. Rev. Lett. 85, 2228 (2000).

[4] M.-O. Mewes, M.R. Andrews, D.M. Kurn, D.S. Durfee, C.G. Townsend, and W. Ketterle, Phys. Rev. Lett. 78, 582 (1997).

[5] S. Inouye, T. Pfau, S. Gupta, A. P. Chikkatur, A. Gorlitz, D. E. Pritchard, and W. Ketterle, Nature 402, 641 (1999).

[6] C. E. Wieman, D. E. Pritchard, and D. J. Wineland, Rev. Mod. Phys. 71, S253 (1999).

[7] S.E. Harris and L.V. Hau, Phys. Rev. Lett. 82, 4611 (1999).

[8] B. Friedrich, R. deCarvalho, J. Kim, D. Patterson, J.D. Weinstein, and J.M. Doyle, J. Chem. Soc., Faraday Trans. 94, 1783 (1998).

[9] R.J. Gordon and S.A. Rice, Annu. Rev. Phys. Chem. 48, 601 (1997).

[10] D. Egorov, J. D. Weinstein, D. Patterson, B. Friedrich, and J. M. Doyle, Phys. Rev. A 63, 030501(R) (2001).

[11] H. Stapelfeldt, H. Sakai, E. Constant, and P. B. Corkum, Phys. Rev. Lett. 79, 2787 (1997).

[12] A. Boca and B. Friedrich, J. Chem. Phys. 112, 3609 (2000).

[13] B. Friedrich and D. R. Herschbach, Nature 353, 412 (1991).

[14] D.R. Herschbach, in Chemical Research - 2000 and Beyond: Challenges and Visions, P. Barkan, Ed. (Oxford University Press, New York, 1998), p. 113.

[15] N. Balakrishnan and A. Dalgarno, Chemical Physics Letters 341, 652 (2001).

[16] C. Zhu, N. Balakrishnan, and A. Dalgarno, Journal of Chemical Physics 115, 1335 (2001); J. L. Bohn, Physical Review A 6305, 2714 (2001); J. L. Bohn, Physical Review A 6203, 2701 (2000); N. Balakrishnan, A. Dalgarno, and R. C. Forrey, Journal of Chemical Physics 113, 621 (2000); J. L. Bohn, Physical Review A 6104, 0702 (2000).

[17] D. DeMille, Physical Review Letters 88, 067901 (2002).

[18] M. Baranov, Y. Kagan, and M. Kagan, JETP Lett., 64, 273 (1996).

[19] M. Houbiers, R. Ferwerda, H. T. C. Stoof, W. I. McAlexander, C. A. Sackett, and R. G. Hulet, Phys. Rev. A 56, 4864 (1997).

[20] M. A. Baranov, M. S. Mar'enko, V. S. Rychkov, and G. V. Shlyapnikov, arXiv:cond-mat 1, 0109437 (2001). 
[21] Y. Kagan, I.A. Vartanyants, and G.V. Shlyapnikov, Sov. Phys. JETP 54, 590 (1981).

[22] L. Santos, G.V. Shlyapnikov, P. Zoller, M. Lewenstein, Phys. Rev. Lett. 85, 1791 (2000).

[23] K. Goral, K. Rzazewski, T. Pfau, Phys. Rev. A 61, 051601 (2000).

[24] K. Goral, L. Santos, M. Lewenstein, Phys. Rev. Lett. 88, 170406 (2002).

[25] M. Baranov, L. Dobrek, K. Goral, L. Santos, M. Lewenstein, arXiv:condmat0201100v1, 8 Jan (2002).

[26] J. T. Bahns, W. C. Stwalley, and P. L. Gould, J. Chem. Phys. 104, 9689 (1996)

[27] J. D. Weinstein, R. deCarvalho, T. Guillet, B. Friedrich, and J. M. Doyle, Nature 395, 148 (1998).

[28] H. L. Bethlem, G. Berden, F. M. H. Crompvoets, R. T. Jongma, A. J. A. van Roij, and G. Meijer, Nature 406, 491 (2000); G. Meijer, private communication.

[29] F. M. H. Crompvoets, H. L. Bethlem, R. T. Jongma, and G. Meijer, Nature 411, 174 (2001).

[30] P. Pillet, C. Drag, and B. L. Tolra, Laser Phys. 11, 480 (2001) and references cited therein.

[31] A.N. Nikolov, J.R. Ensher, E.E. Eyler, H. Wang, W.C. Stwalley, and P.L. Gould, Phys. Rev. Lett. 84, 246 (2000) and references cited therein.

[32] R. Wynar, R. Freeland, D. Han, C. Ryu, and D. Heinzen, Science 287, 1016 (2000).

[33] M. Gupta and D. Herschbach, J. Phys. Chem. A 105, 1626 (2001).

[34] B. Friedrich, Phys. Rev. A 61, 5403 (2000).

[35] B. Friedrich and D. Herschbach, Phys. Rev. Lett. 74, 4623 (1995).

[36] R. deCarvalho, J. M. Doyle, B. Friedrich, T. Guillet, J. Kim, D. Patterson, and J. D. Weinstein, Eur. Phys. J. D 7, 289 (1999).

[37] D. Egorov, T. Lahaye, W. Schoellkopf, B. Friedrich, J.M. Doyle, Phys. Rev. A 66, 043401 (2002).

[38] R. deCarvalho, C. Hancox, J.M. Doyle, JOSA B, to be published.

[39] J.M. Doyle, B. Friedrich, J. Kim, and D. Patterson, Phys. Rev. A 52, R2515 (1995).

[40] J. Weinstein, R. deCarvalho, T. Guillet, B. Friedrich, and J.M. Doyle, Nature 395, 148 (1998).

[41] L.E. Berg and L. Klyning, Physica Scripta 10, 331 (1974).

[42] H. Martin, J. Mol. Spect. 108, 66, (1984).

[43] T. Leininger and G. Jeung, J. Chem. Phys. 103, 3942 (1995).

[44] B. Friedrich, R. deCarvalho, J.D. Weinstein, and J.M. Doyle, J. Chem. Phys. 110, 2376 (1999).

[45] J. D. Weinstein, R. deCarvalho, K. Amar, A. Boca, B. C. Odom, B. Friedrich, and J. M. Doyle, J. Chem. Phys. 109, 2656 (1998). 
[46] G. Scoles, Atomic and molecular beam methods. (Oxford University Press, New York, 1988).

[47] H. Pauly, Atom, molecule, and cluster beams. (Springer, Berlin, 2000).

[48] Infrared Laboratories HDL-5

[49] $1.7 \mathrm{~mm}$ inner diameter, $50 \mathrm{~cm}$ long

[50] The pressure in the cell is monitored by a Pirani gauge (Grainville-Phillips Convectron 250), connected to the cell via a straight stainless steel tube, $45 \mathrm{~cm}$ long and of a $1.9 \mathrm{~mm}$ inner diameter. The gauge is at room temperature, so a difference between the pressure reading and the actual pressure in the cryogenic cell can exist, see T. R. Roberts and S. G. Sydoriak, Phys. Rev. 102, 304 (1956). Also, helium gas flow may affect the pressure reading. By performing a number of measurements, such as drop of pressure in known-volume gas supply vessel and pressure measurements for different orifice openings with a ruthenium oxide sensor suspended inside the cell, we established that these effects fall within the absolute calibration uncertainty of the pressure gauge, and have no effect on its relative accuracy.

[51] Thin copper cups with 20/40 mesh coconut activated charcoal (Supelco \#10275) attached by epoxy

[52] D. W. Sedgley, A. G. Tobin, T. H. Batzer, and W. R. Call, J. Vac. Sci. Technol. A 5, 2572 (1987).

[53] M. R. Walkiewicz, P. J. Fox, and R. E. Scholten, Rev. Sci. Instrum. 71, 3342 (2000).

[54] E. Arimondo, M. Inguscio, and P. Violino, Rev. Mod. Phys. 49, 31 (1977)

[55] G. P. Barwood, P. Gill, and W. R. C. Rowley, Appl. Phys. B 53, 142 (1991).

[56] M. V. Romalis, E. Miron, and G. D. Cates, Phys. Rev. A 56, 4569 (1997).

[57] A. Hatakeyama, K. Oe, K. Ota, S. Hara, J. Arai, T. Yabuzaki, and A. R. Young, Phys. Rev. Lett. 84, 1407 (2000).

[58] A. Einstein, Phys. Rev. A 00, 0101 (1911).

[59] S. Timoshenko and S. Woinowsky-Krieger, Theory of Plates and Shells, McGrawHill, New York (1959). 